\title{
Recto-Vesical Pouch
}

National Cancer Institute

\section{Source}

National Cancer Institute. Recto-Vesical Pouch. NCI Thesaurus. Code C33448.

The peritoneal reflection between the urinary bladder and the rectum in males. 